\title{
LEVERAGING TECHNOLOGY BY DIGITALIZATION USING "I REPORT APP" FOR SAFETY AT CONSTRUCTION SITES
}

\author{
Rishikesh Ahirrao ${ }^{1}$
}

\begin{abstract}
Construction industry is highly unorganized in most developing countries and prone to major risks and safety non-conformances. Identification of unsafe conditions and unsafe acts at construction sites is a huge challenge due to less participation from stakeholders, reluctance in filling up the manual formats and inconsistency in hazard reporting, recording and timely closure of safety issues resulting in various wastes affecting organization's Occupational Health and Safety (OHS) performance. Digitization has its issues such as unavailability of a user-friendly systems at construction sites etc. This slows down the safety improvements and risk mitigation initiatives.

In order to address the operational challenges of delivering "Safe projects with the aim of "zero accidents" the organisation took up the task of exploring Information Technology (IT) to create an App suitable to all stakeholders and which would provide a platform for quick redressal of safety concerns and improvement of existing safety processes. The paper explains how the mobile App I-Report was developed by collaborating with all internal stakeholders and how it succeeded in improving the safety performance of the organisation. How digitization helped create safe work sites, reduce accidents and incidents by continuous improvements of safety processes based on IT enabled data analytics is answered in this paper.
\end{abstract}

\section{KEYWORDS}

Lean App, Safety, hand held assistant, hazard identification and reporting.

\section{INTRODUCTION}

Hazard identification is fundamental to construction safety management and it is often the unidentified hazards that present the most unmanageable risks. Maximum hazard identification levels were found to be $89.9 \%$ for a construction project within the nuclear industry, $72.8 \%$ for a project within the railway industry, and $66.5 \%$ for a project within both the railway and general construction industry sector. The results indicate that hazard identification levels are far from ideal and an IT tool for construction project safety management is helpful (Gregory Carter and Simon D. Smith, 2006). BIM-based fall

\footnotetext{
${ }^{1}$ OHS Head-Godrej Construction, Godrej \& Boyce Mfg. Co Ltd, Mumbai, India; +91-9833591597;
} rma@godrej.com 
hazard identification and prevention in construction safety planning with only limited automation in modelling and planning safety processes has been exploited so far (Kristina Sulankivib, Ilkka Romoc Charles M.Eastmand, Jochen Teizere (2015).Also, Javier Irizarry(2012) explores Drone Technology as Safety Inspection Tools while pointing out that the construction industry lags behind many others in the rate of adoption of cutting edge technologies in the area of safety management. Advances in information technology could provide great benefits to this important aspect of construction operations and innovative use of these tools could result in safer jobsites. A comprehensive study by Jochen Teizer (2015) evaluates the capability of the developed Self-Monitoring Alert and Reporting Technology for Hazard Avoidance and Training (Smart Hat) technology, a novel battery-free sensing and communication prototype that also provides alerts in realtime when hazardous proximity conditions are present between heavy construction equipment and ground workers. The construction industry generally struggles to achieve the psychological safety needed for people to speak up. And that same challenge applies beyond safety, to other improvements in work processes, to promoting experimentation in short, to achieving a lean culture. (Howell, G., Ballard, G. and Demirkesen, S,July 2017).

Construction sites are messy and chaotic places with materials, equipment, vehicles and people all over the place in a constant state of motion. The intrinsic nature of construction activities gives rise to safety hazards galore. At construction sites, multiple trades work simultaneously such as civil work, plumbing, carpentry, tiling, electrical installations etc. and that too under pressure to meet the deadlines, results in a "perfect storm" giving rise to dangerous, life threatening hazards at its core.

The paper demonstrates how the efficacy of development and implementation of the Application were enhanced using a Lean Approach adopting stakeholder's collaboration, innovative thinking, continuous improvement and Value Stream Improvement. The paper also shows statistics of improvements in safety achieved by using the Lean based App.

\section{CHALLENGES FACED BY THE ORGANIZATIONS FOR HAZARD REPORTING:}

The organisation was faced with many existing challenges in hazard reporting at its various construction sites. Listed below are some of the key challenges to safety as felt by various stakeholders:

- Frequent changing workforce at construction projects

- Real time information about hazard available only with the individual observing hazard

- Less participation from all stakeholders

- System inaccessibility leading to reluctance in filling up the formats etc.

- Reporting done only when on duty.

- Non-availability of any user-friendly system at sites.

- Inconsistency in Hazard reports received

- Delayed recording and closure of safety issues

- System generated Trends Analysis not available

- Cumbersome to make available trends and their analysis for safety improvements. 


\section{OTHER METHODS OF SAFETY HAZARDS REPORTING PRIOR TO APP}

The organisation explored alternate available platforms for Hazard Reporting prior to going Digital with App Development such as:

- Reporting Hazards \& Incidents on printed formats.

- Online system to report Hazards \& Incidents - The Speed-flow System

- Reporting on social media through WhatsApp Groups.

- Monthly reports to Senior Management using Power Point presentations.

- Telephone calls to team, vendors and other stakeholders involved about prevalent hazards.

However, the challenges of reliable reporting were not addressed by the processes mentioned above.

\section{RATIONALE FOR ADOPTING A MOBILE APP FOR SAFETY REPORTING:}

- No formal training requirement: The world has seen lot of increase in the mobile users who use variety of apps for online shopping, banking apps, etc. The main advantage of a Mobile App is that there is no formal training required for usage.

- Ease of Hazard Reporting: The organization wanted to identify all hazards however insignificant and ensure proper capturing of the hazard transmittals to the authorities concerned for prompt corrective action, analysis and development of preventive action practices by well mapped processes. The App had to be a perfect mix of an automated, streamlined, paperless process that made reporting hazards at sites instantaneous, traceable and easier.

- Acts as a Lean Tool for ensuring Psychological Safety with Stakeholder Empowerment: The App was named "I Report" App and the prime requirement was to boost psychological safety to empower construction workers, supervisors, contractors, and management staff by encouraging hazard reporting and their immediate closure thus creating a medium for fast hazard closure using the user friendly App.

- Help address Lead Indicators versus Lag Indicators for futuristic safety improvements across construction activities: The hazards provided lead indicators as reported on the App which could be used modifying existing safety to eliminate any incidents or unsafe conditions or acts.

research methodology and stages of app development: 


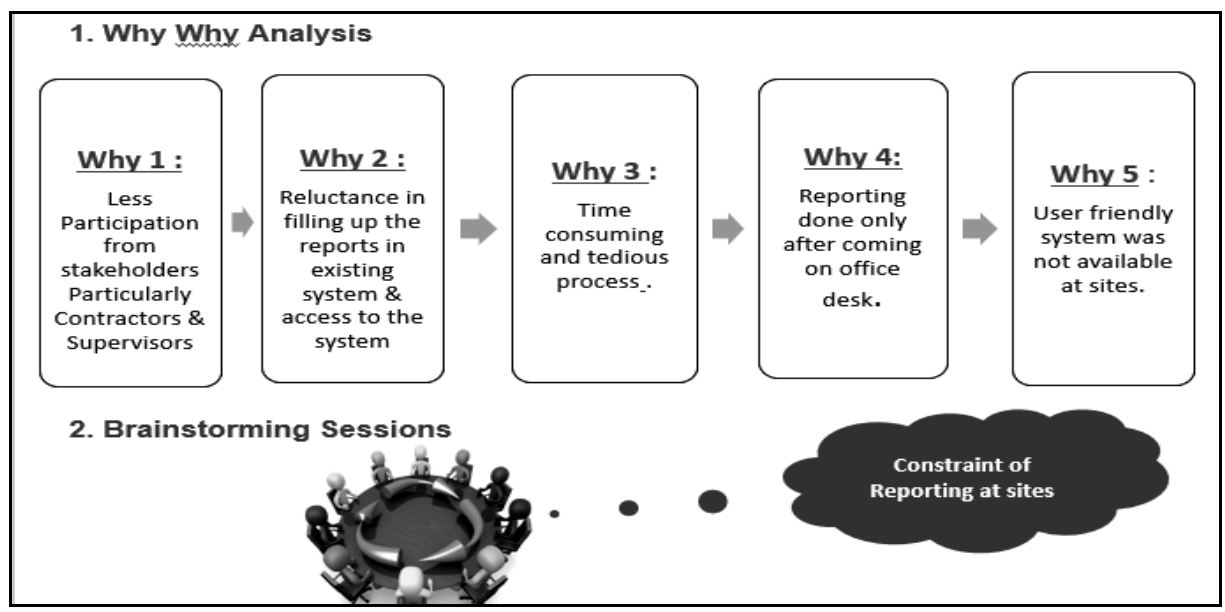

Figure 1: Root Cause Analysis

\section{STEP 1: Capturing Needs and Expectations of the Application:}

The organisation conducted a Root Cause Analysis along with a series of brainstorming sessions of Senior Management and stakeholders and a need was felt to have efficient system leveraging technology for timely actions on hazards. Severe constraints such as scattered site locations and deficiencies of existing system as described previously were identified.

\section{STEP 2: Adoption of a Focussed Approach for Lean App Development:}

a. Modification of existing Online "Speed flow System"

Despite modification in the existing online system, there were certain drawbacks such as:

Limited Accessibility: Accessible only to the management and other stakeholders were not in a position to report hazards.

Lack of Pictorial or visual information: No feature for attaching the photograph of the hazard in the system for clarity of the person taking action for hazard closure.

Limited safety empowerment: Contractors and other vendors comprising of more than $80 \%$ of the work force who working at site were did not have any access to the system.

Delayed Hazard Reporting: Real time reporting from site / area of safety violation not possible.

Reports/Trends Generation Ability: No reports were generated through the system.

\section{b. Collection of Reports from Stakeholders on monthly basis:}

In the interim period during the development of the Lean App, in order to ensure complete stakeholder involvement, all the teams were requested to compile and share reports for incidents, hazards, near misses at various construction locations on manual formats. 


\section{c. Exploration other Digital Platforms:}

The collection of reports, their analysis was cumbersome and there was inconsistency in the reporting as the hazards were not getting captured real time nor was their closure traceable. Also, the information available by the reports were limited to the centralized Safety Team. This made the horizontal deployment and sustainability of good safety practices a huge challenge.

The organisation decided increase the data availability to create trust and transparency by leveraging the existing Social Media platforms like WhatsApp in an attempt to bridge the gap for better communication management .

\section{d. The Design, Development and Launch of the Lean App:}

Finally, based the shortcomings of the above initiatives made the organisation design the I report App and it was developed internally by the organisations IT department. All that "I-Report" required was a smart phone, mental alertness and a sense of responsibility to report the hazard. It was ensured that interface of the app remained simple and intuitive it did not require any extensive training. Supporting the app at the back-end was the organisations database where all the reported hazards were captured and analysed for initiating corrective measures to avoid such unsafe conditions in the future.

This APP is not just a smart way of reporting hazards, incidents or get data metrics, but is a start of new safety revolution Since inception of this App, reporting and closure of hazards at the organisations construction sites has improved significantly.

This is one of the most effective safety communication system and is likely to become the best practice of not only of the organization but also industry wide.

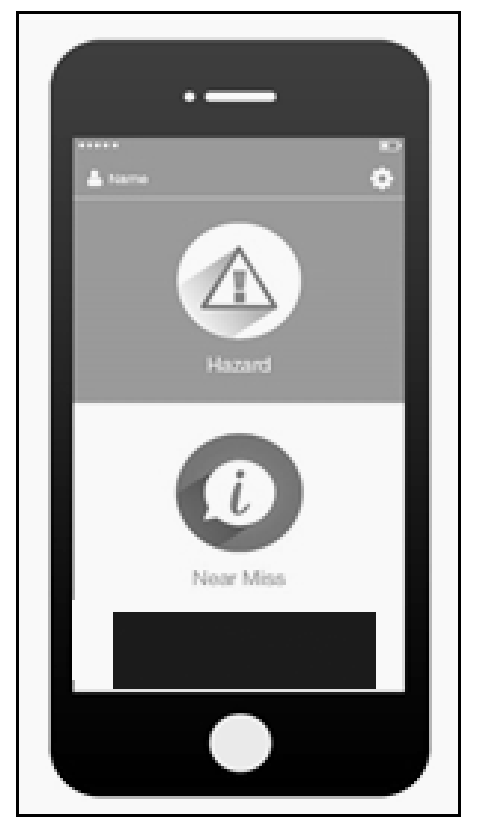

Figure 2: App Development Stages 


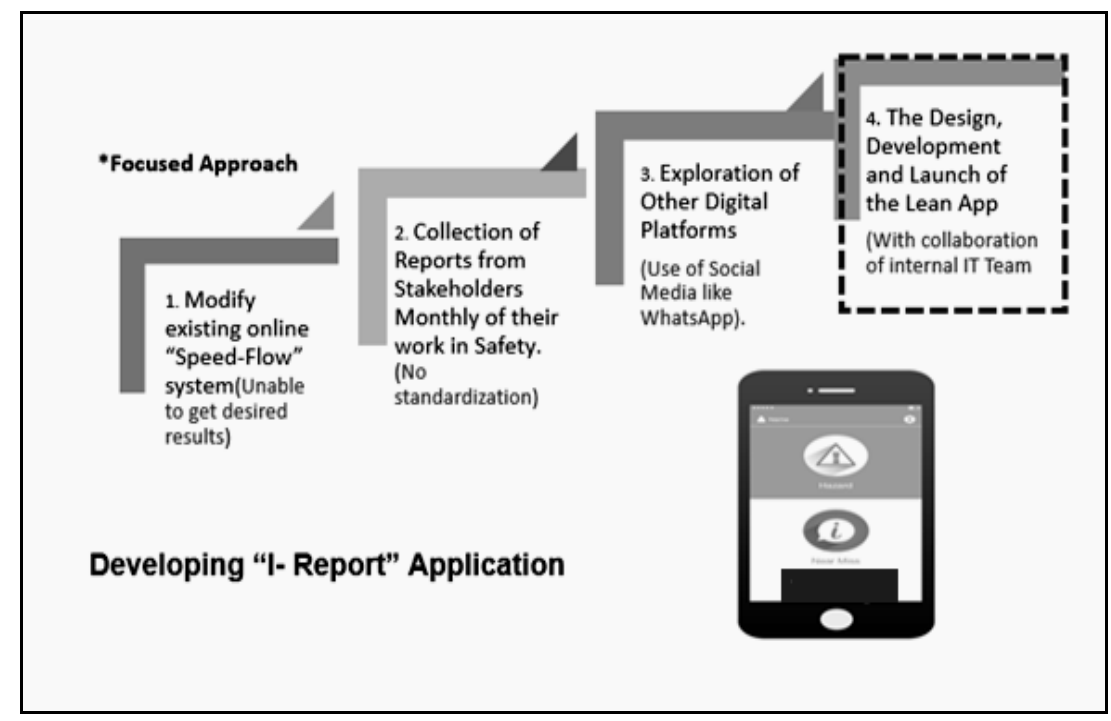

A Smart Way to report Hazards \& Incidents

Figure 3: "The I Report App"

\section{Uniqueness of The I Report Application:}

First Application in India for hazard reporting

Customised to suit stakeholder's expectations.

Low Cost for design, development and maintenance

This App is a perfect mix of an automated, streamlined, paperless process that makes reporting hazards at sites instantaneous, traceable and easier.

Every hazard has a unique no generated after being reported.

Supported by a strong database system.

\section{Benefits of "I - REPORT" APP}

Real time hazard reporting

Boosts Psychological Safety

Futuristic in its approach towards Safe Working Sites

Fast Recording and Hazard Closure

Easy Recording and Closing Near Miss Incidents

Waste Minimization (Time, Cost)

Effective communication management across team

Horizontal Deployment for best safety practices

Consistency and Standardization in reporting of hazards.

Easy Trends analysis with minimal human intervention

Accountability and responsibility in line managers

Availability of safety trends and their analysis activity wise, location wise etc. 
What the "I report" App facilitates:

Flow Chart aiming for "continuous improvement"

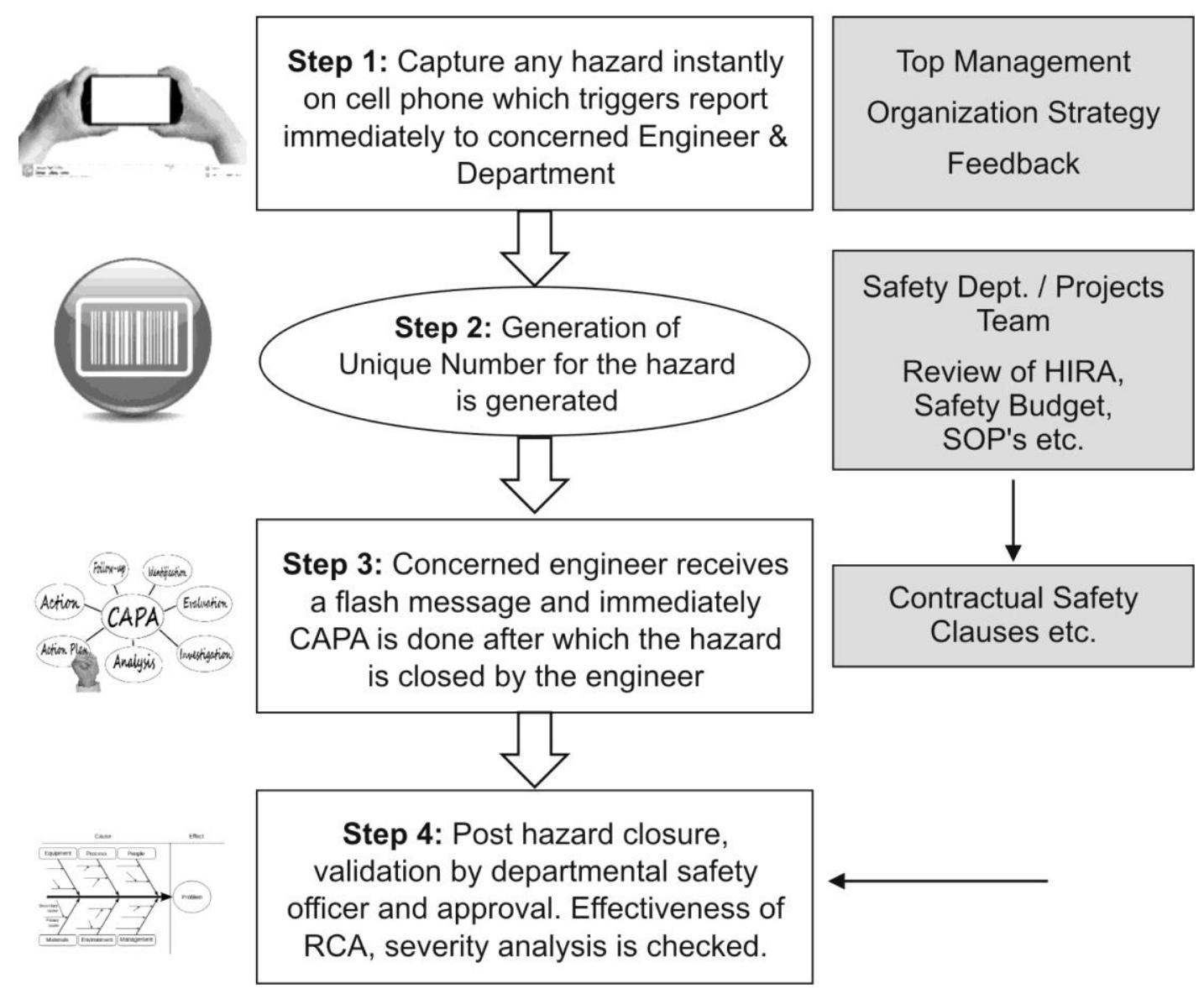

Flow Process for Hazard Report and closure through the "I Report App"

\section{SUCCESS MEASURES AND RESULTS}

\section{TANGIBLE BENEFITS}

Hazard Identification increased Multifold: The amount of reporting the hazards has been improved, earlier the hazards in the year 2015-2016 was 1045 and post successful launch and use the hazards reported in the year 2017-2018 were.

Root Cause Analysis of all Hazards: A Detailed Root Cause Analysis (RCA) is being done for all the hazards identified and the results categorised for further actions.

Going Green \& Paper less Records: Post launch of the I report app ,100 percent hazards were reported on the app, which helped us in our go green and save paper.

Trend Analysis for futuristic action on Safety Focus Areas: Post report generation shared with teams, stakeholders and Top Management trend analysis for causes of hazards, Senior Management could easily identify its Focus Areas for safety. 
Saves Cost of Accident: Hazard reporting, closure, analysis drive process improvements and this reduces the chances of accident and the cost of the accident direct cost and indirect cost is saved.

Continuous improvement of Safety Processes and regular updating of Hazard Identification and Risk assessment (HIRA) Register:

Post the I report App the organisation has updated and added more than 150 safety processes and HIRA register updated on quarterly basis as compared to yearly basis prior to App launch. Proactive and futuristic approach rather than reactive approach has resulted.

\section{INTANGIBLE BENEFITS}

Improved Quality of reporting: The quality of reporting the hazards also has improved post "I - Report" launch and Implementation.

Enhanced Responsibility and Accountability: The I report application makes accountable the Plant Managers, Project Managers to close the hazards reported within the specified time (72 hours maximum). Aging analysis of hazard closure drives performance of Project Managers, Safety Officer and their teams.

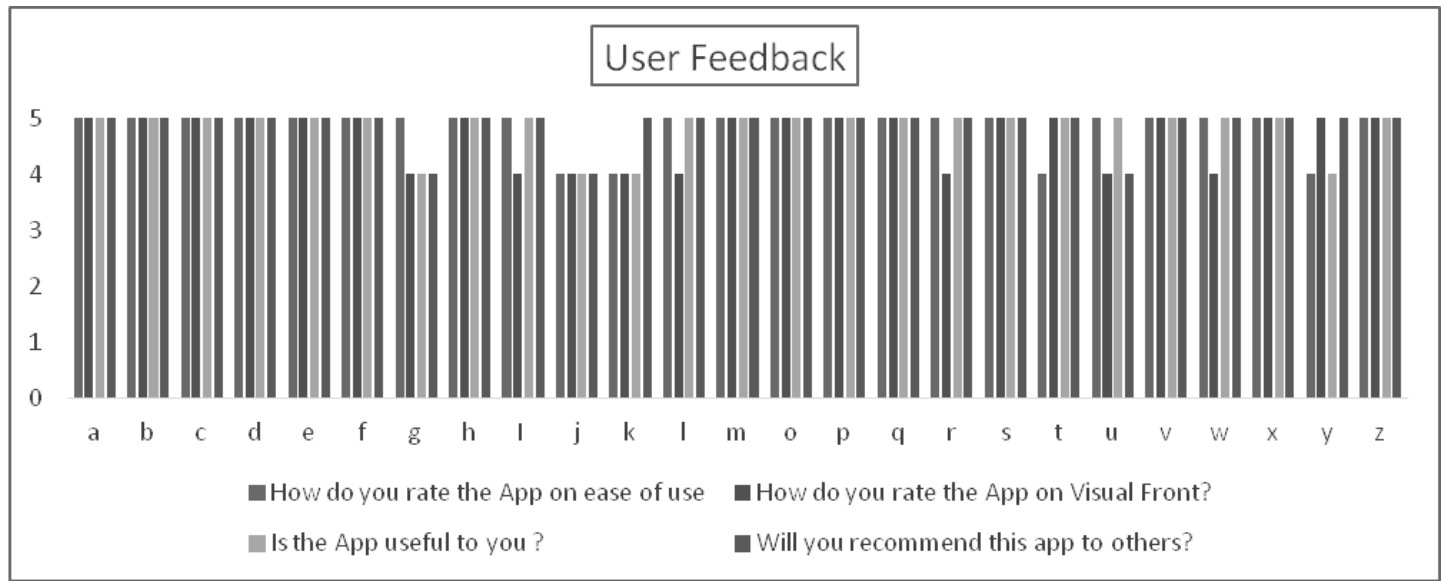

Figure 4: Graph indicating stakeholders feedback

FEEDBACK (VOICE OF THE STAKEHOLDERS) OF THE APP:

\section{Customer A}

"I report App" - Great Initiative by the organisation to attain the goal of Zero Incident.

\section{Customer B}

User Friendly App, now my team can capture all the hazards without going to desk to report it and saving a lot of time

\section{Customer C}

Great App, for safety, looking forward to using new apps reducing work load 


\section{Customer D}

Good Initiative by the organisation and extending the use to its vendors and contractors.

\section{CONCLUSION}

Adopting a safety philosophy emphasizing "Respect to Human Life "which improves morale of all stakeholders ultimately leads to improved business performance. Apps such as "I report" facilitate collaboration for creating accident free sites by providing a platform for quick hazard reporting and their immediate closure thereby reducing probable accidents which can cause human losses, productivity losses ultimately affecting overall project performance and hampering brand image. The whole purpose of bringing technology was to improve psychological safety by empowering every stakeholder working for the company with the ultimate aim to make construction sites in the organisation safe and motivating workplaces.

Diligent Root Cause Analysis and Corrective and Preventive Action for repeated hazards leads to elimination of unsafe conditions by driving change initiation activities in conventional operational procedures or workflow methodologies followed in the industry thereby setting a benchmark by $\mathrm{f}$ continual improvement enabling the organisation set standards in safety to the nation.

\section{REFERENCES:}

Book on Lean Construction by LUIS ALARCON.

Choonsung Shin, Jin-Hyuk Hong, Anind K. Dey (2015) -Understanding and prediction of mobile application usage for smart phones.

Edward J. Jaselskis, Stuart D. Anderson, Jeffrey S. Russell - (1996)- Strategies for Achieving Excellence in Construction Safety Performance.

Gregory Carter and Simon D. Smith - (2006) Safety Hazard Identification on Construction Projects

Howell, G., Ballard, G. and Demirkesen, S- (2017) Why Lean Projects are safer?

Jochen Teizer (2015) - Wearable, wireless identification sensing platform: SelfMonitoring Alert and Reporting Technology for Hazard Avoidance and Training (SmartHat)

Kristiina Sulankivib, Ilkka Romoc Charles; M. Eastmand, Jochen Teizere- (2015) - BIMbased fall hazard identification and prevention in construction safety planning.

\section{Referred Websites:}

http://blog.nielsen.com/nielsenwire/online_mobile/thestate-of-mobile-apps/

http://www.distimo.com/blog/2011/04/

http://www.research2guidance.com 